\title{
Assessment of inter- and intra-observer reliability in the determination of radiographic version and inclination of the cup in metal-on-metal hip resurfacing
}

\author{
Aleksi Reito • Timo Puolakka • Antti Paakkala • \\ Jorma Pajamäki
}

Received: 25 May 2011 / Accepted: 13 July 2011 / Published online: 12 August 2011

(C) Springer-Verlag 2011

\begin{abstract}
Purpose Determination of the cup orientation after metal-on-metal hip resurfacing may provide important information in the postoperative follow-up. We present a mathematical method based on a previously described approach to assess the version and inclination of the cup in the metal-on-metal bearing without a separate software computation from plain radiographs. The aim of the study was to assess the intra- and inter-observer reliability of this method.

Methods Calculation of version and inclination were done twice for 20 hip resurfacings by four observers. Intraobserver reliability was estimated by mean error and correlation of the two sets of measurement for version and inclination. Bland-Altman plots, intra-class coefficient and mean error were used to assess the inter-observer reliability of the measurements.

Results Intra-observer correlation for version measurement ranged from 0.74 to 0.94. Correlation for inclination varied between 0.94 and 0.97. Upper and lower limits of agreement in Bland-Altman plots for version measurements between observers ranged from 4.1 to 7.2 degrees and from -3.2 to -8.3 degrees, respectively. For inclination measurements the upper and lower limits ranged from 3.1 to 5.3 degrees and from -2.7 to -6.0 degrees.
\end{abstract}

A. Reito $\cdot$ T. Puolakka $\cdot$ J. Pajamäki $(\bowtie)$

Coxa Ltd, Hospital for Joint Replacement,

Tampere, Finland

e-mail: j.pajamaki@coxa.fi

A. Reito

e-mail: aleksi.reito@uta.fi

A. Paakkala

Medical Imaging Centre, Tampere University Hospital,

Tampere, Finland
Conclusions Mean errors, correlation coefficients and 95\% limits of agreement were on an acceptable level. We believe that this method is applicable for clinical use.

\section{Introduction}

Postoperative radiographs are an important tool in the routine follow-up of the hip arthroplasties. In addition to qualitative analysis of the postoperative radiographs, quantitative measurements may also provide important information regarding the performance of the prosthesis. In total hip arthroplasties (THA), component malposition, i.e. excess version or inclination of the cup, is associated with impingement and dislocations [1]. At present it is well recognised that in the metal-on-metal hip resurfacings (MMHR) the wear related problems are greatly associated with excessive inclination leading to reduced articular surface and edge-loading on the acetabular side [2]. In some cases also the version of the cup has an influence on wear [3, 4].

Several papers have been published presenting different methods of measuring the cup orientation in THA from plain anteroposterior (AP) radiographs [5-11]. All methods are based on different calculations with the aid of the ellipse formed by the cup opening. In hip resurfacing this presents a problem since the components are all metal and the cup opening cannot be clearly visualised (Fig. 1). Most of the methods presented require the presence of the metal ring in the polyethylene cup. The method proposed by Hassan et al. is the only method suitable for all-metal arthroplasties [11]. It was originally devised for metal-backed metal-onpolyethylene THAs which do not contain a metallic indicator ring.

In numerous hip resurfacing studies assessing the cup version, Ein Bild Roentgen Analysis (EBRA) has been used 
Fig. 1 a Birmingham hip resurfacing (BHR) components in neutral position showing both component corners. b BHR cup anteverted overlapping the femoral component. c BHR dysplasia cup in normal position. $\mathbf{d}$ BHR dysplasia cup anteverted overlapping the femoral component
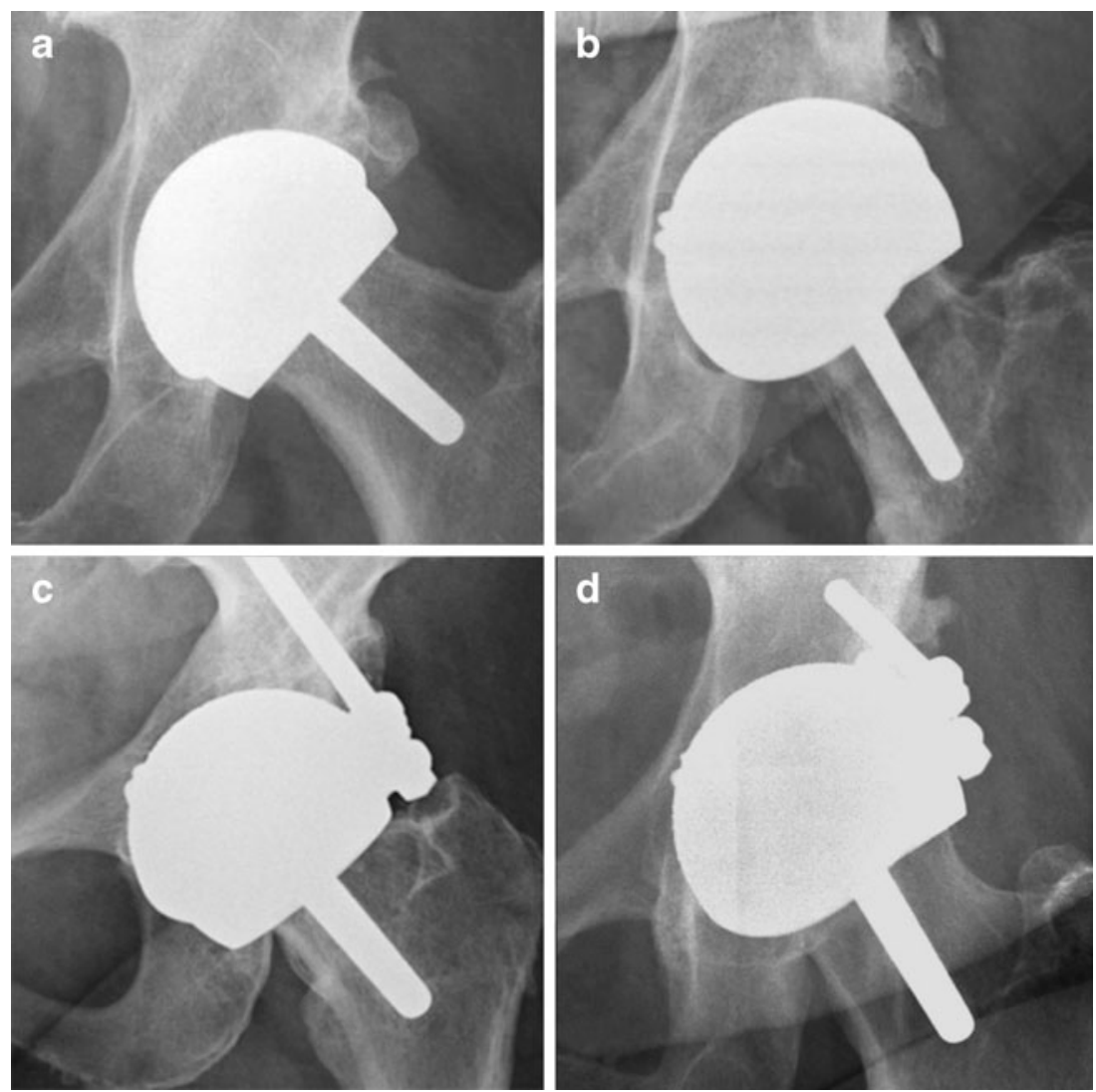

in calculations [4, 12-14]. In one study the method by Widmer was used whereas Ball et al. used the axiolateral version proposed by Yao [15-17]. Inclination of the cup is straightforward to calculate from plain radiographs. Occasionally problems may occur when there is overlapping of the shadows of the components or there is a dysplasia cup distorting the reference points. However, in clinical use the calculation of version is the most challenging assessment (Fig. 1).

We developed the method by Hassan et al. to calculate the version of the cup in MMHR [11]. The aim of this study was to define the inter- and intra-observer reliability and estimate the usefulness of this method in the assessment of the cup orientation in MMHR.

\section{Materials and methods}

Finding the end points of the major semi axis of the ellipse formed by the cup opening can be simply done by drawing a circle which is continuous to the outline of the cup (Fig. 2). The points where the cup outline diverge from the circle represents the end points of the major semi axis of the ellipse. The origin of the ellipse is the midpoint of the major semi axis. If the cup is hemispherical this point is equal to centre of rotation of the cup (RC). Then an arbitrary line is drawn perpendicular to the semi axis as described by Hassan et al. and in the case of MMHR the ideal point is to draw it through the point where the shadows of the cup and femur component cross. If the cup

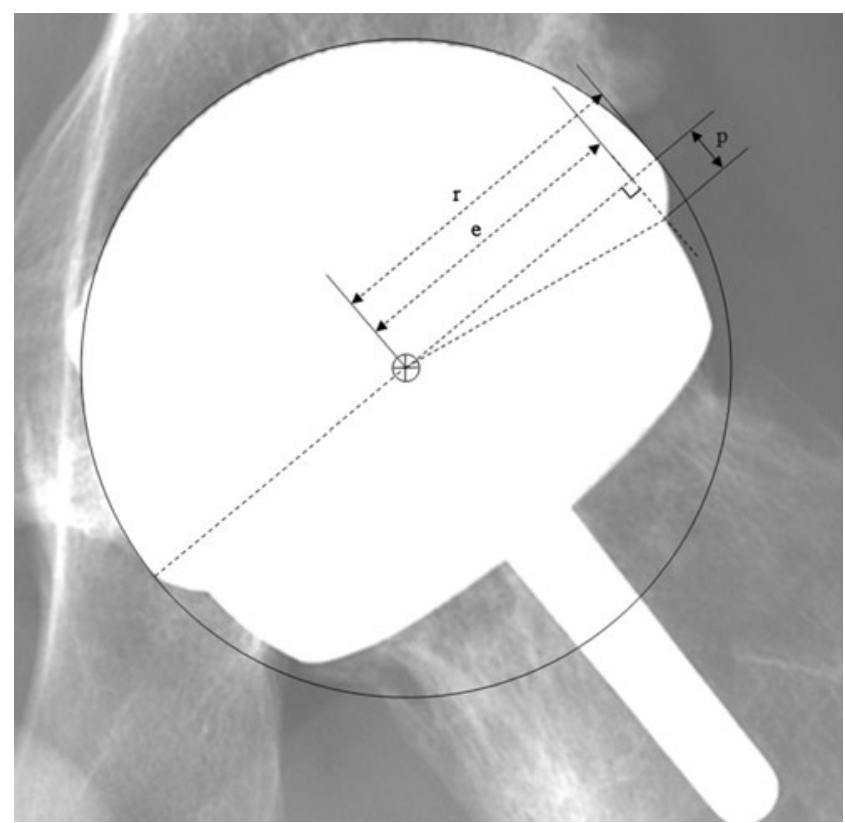

Fig. 2 Scheme of drawings made to assess the cup orientation in metal-on-metal hip resurfacings (MMHR) 
component overlaps the femoral component the perpendicular line can be drawn through any point (Fig. 1b, d). Then the distances $r, e$ and $p$ are measured (Fig. 2). (Ante)version can be calculated as

Versio $=\arcsin \left(\frac{\mathrm{p}}{\sqrt{\mathrm{r}^{2}-\mathrm{e}^{2}}}\right)$

Taking $e^{2}$ as a common factor the expression inside the brackets of the formula may be derived to<smiles>I[C](I)I</smiles>

Thus the version is defined by two variables and a simple chart may be formed to assess the version (Fig. 3).
Exact calibration of the image is not necessary since the calculation is based on the ratios of the lengths.

Inclination of the cup is the angle between the major semi-axis of the ellipse formed by the cup opening and the horizontal reference line. Lines $r, e$ and all lines parallel to them represent the major semi-axis of the ellipse (Fig. 2).

We randomly selected 20 hips from our previous study [18]. Four different observers calculated the version and inclination from every hip twice with a minimum one-week interval. The first observer (observer A) was the person who developed the formula and who also is familiar with the computing software. The method was shortly introduced to three other observers. Two (observers B and C) were senior orthopaedic surgeons who were familiar with the computing software. The third observer (observer D) was not familiar with the computing software. MediCAD
Fig. 3 Version defined by ratio of $p$ and $e$ and ratio of $r$ and $e$ $\mathrm{r} / \mathrm{e}$

\begin{tabular}{|c|c|c|c|c|c|c|c|c|c|c|c|c|c|c|c|c|}
\hline & 1,05 & 1,06 & 1,07 & 1,08 & 1,09 & 1,1 & 1,11 & 1,12 & 1,13 & 1,14 & 1,15 & 1,16 & 1,17 & 1,18 & 1,19 & 1,2 \\
\hline 0,01 & 1,8 & 1,6 & 1,5 & 1,4 & 1,3 & 1,3 & 1,2 & 1,1 & 1,1 & 1,0 & 1,0 & 1,0 & 0,9 & 0,9 & 0,9 & 0,9 \\
\hline 0,02 & 3,6 & 3,3 & 3,0 & 2,8 & 2,6 & 2,5 & 2,4 & 2,3 & 2,2 & 2,1 & 2,0 & 1,9 & 1,9 & 1,8 & 1,8 & 1,7 \\
\hline 0,03 & 5,4 & 4,9 & 4,5 & 4,2 & 4,0 & 3,8 & 3,6 & 3,4 & 3,3 & 3,1 & 3,0 & 2,9 & 2,8 & 2,7 & 2,7 & 2,6 \\
\hline 0,04 & 7,2 & 6,5 & 6,0 & 5,6 & 5,3 & 5,0 & 4,8 & 4,5 & 4,4 & 4,2 & 4,0 & 3,9 & 3,8 & 3,7 & 3,6 & 3,5 \\
\hline 0,05 & 9,0 & 8,2 & 7,5 & 7,0 & 6,6 & 6,3 & 6,0 & 5,7 & 5,5 & 5,2 & 5,1 & 4,9 & 4,7 & 4,6 & 4,4 & 4,3 \\
\hline 0,06 & 10,8 & 9,8 & 9,1 & 8,5 & 8,0 & 7,5 & 7,2 & 6,8 & 6,5 & 6,3 & 6,1 & 5,9 & 5,7 & 5,5 & 5,3 & 5,2 \\
\hline 0,07 & 12,6 & 11,5 & 10,6 & 9,9 & 9,3 & 8,8 & 8,4 & 8,0 & 7,6 & 7,3 & 7,1 & 6,8 & 6,6 & 6,4 & 6,2 & 6,1 \\
\hline 0,08 & 14,5 & 13,2 & 12,1 & 11,3 & 10,6 & 10,1 & 9,6 & 9,1 & 8,7 & 8,4 & 8,1 & 7,8 & 7,6 & 7,3 & 7,1 & 6,9 \\
\hline 0,09 & 16,3 & 14,8 & 13,7 & 12,7 & 12,0 & 11,3 & 10,8 & 10,3 & 9,8 & 9,5 & 9,1 & 8,8 & 8,5 & 8,3 & 8,0 & 7,8 \\
\hline 0,1 & 18,2 & 16,5 & 15,2 & 14,2 & 13,3 & 12,6 & 12,0 & 11,4 & 11,0 & 10,5 & 10,1 & 9,8 & 9,5 & 9,2 & 8,9 & 8,7 \\
\hline 0,11 & 20,1 & 18,2 & 16,8 & 15,6 & 14,7 & 13,9 & 13,2 & 12,6 & 12,1 & 11,6 & 11,2 & 10,8 & 10,4 & 10,1 & 9,8 & 9,5 \\
\hline 0,12 & 22,0 & 20,0 & 18,4 & 17,1 & 16,1 & 15,2 & 14,4 & 13,8 & 13,2 & 12,7 & 12,2 & 11,8 & 11,4 & 11,0 & 10,7 & 10,4 \\
\hline 0,13 & 24,0 & 21,7 & 20,0 & 18,6 & 17,4 & 16,5 & 15,7 & 14,9 & 14,3 & 13,7 & 13,2 & 12,8 & 12,4 & 12,0 & 11,6 & 11,3 \\
\hline 0,14 & 25,9 & 23,5 & 21,6 & 20,1 & 18,8 & 17,8 & 16,9 & 16,1 & 15,4 & 14,8 & 14,3 & 13,8 & 13,3 & 12,9 & 12,5 & 12,2 \\
\hline 0,15 & 27,9 & 25,3 & 23,2 & 21,6 & 20,2 & 19,1 & 18,1 & 17,3 & 16,6 & 15,9 & 15,3 & 14,8 & 14,3 & 13,9 & 13,4 & 13,1 \\
\hline 0,16 & 30,0 & 27,1 & 24,9 & 23,1 & 21,6 & 20,4 & 19,4 & 18,5 & 17,7 & 17,0 & 16,4 & 15,8 & 15,3 & 14,8 & 14,4 & 14,0 \\
\hline 0,17 & 32,1 & 28,9 & 26,5 & 24,6 & 23,1 & 21,8 & 20,7 & 19,7 & 18,8 & 18,1 & 17,4 & 16,8 & 16,3 & 15,7 & 15,3 & 14,8 \\
\hline 0,18 & 34,2 & 30,8 & 28,2 & 26,2 & 24,5 & 23,1 & 21,9 & 20,9 & 20,0 & 19,2 & 18,5 & 17,8 & 17,2 & 16,7 & 16,2 & 15,7 \\
\hline 0,19 & 36,4 & 32,7 & 29,9 & 27,8 & 26,0 & 24,5 & 23,2 & 22,1 & 21,2 & 20,3 & 19,5 & 18,9 & 18,2 & 17,7 & 17,1 & 16,6 \\
\hline 0,2 & 38,7 & 34,7 & 31,7 & 29,4 & 27,5 & 25,9 & 24,5 & 23,4 & 22,3 & 21,4 & 20,6 & 19,9 & 19,2 & 18,6 & 18,1 & 17,5 \\
\hline 0,21 & 41,0 & 36,7 & 33,5 & 31,0 & 29,0 & 27,3 & 25,8 & 24,6 & 23,5 & 22,6 & 21,7 & 20,9 & 20,2 & 19,6 & 19,0 & 18,5 \\
\hline 0,22 & 43,4 & 38,7 & 35,3 & 32,6 & 30,5 & 28,7 & 27,2 & 25,9 & 24,7 & 23,7 & 22,8 & 22,0 & 21,2 & 20,6 & 19,9 & 19,4 \\
\hline 0,23 & 45,9 & 40,9 & 37,2 & 34,3 & 32,0 & 30,1 & 28,5 & 27,1 & 25,9 & 24,8 & 23,9 & 23,0 & 22,3 & 21,5 & 20,9 & 20,3 \\
\hline 0,24 & 48,6 & 43,1 & 39,1 & 36,0 & 33,6 & 31,6 & 29,9 & 28,4 & 27,1 & 26,0 & 25,0 & 24,1 & 23,3 & 22,5 & 21,8 & 21,2 \\
\hline 0,25 & 51,3 & 45,3 & 41,1 & 37,8 & 35,2 & 33,1 & 31,3 & 29,7 & 28,4 & 27,2 & 26,1 & 25,2 & 24,3 & 23,5 & 22,8 & 22,1 \\
\hline 0,26 & 54,3 & 47,7 & 43,1 & 39,6 & 36,8 & 34,6 & 32,7 & 31,0 & 29,6 & 28,4 & 27,2 & 26,2 & 25,3 & 24,5 & 23,8 & 23,1 \\
\hline 0,27 & 57,5 & 50,2 & 45,2 & 41,4 & 38,5 & 36,1 & 34,1 & 32,4 & 30,9 & 29,6 & 28,4 & 27,3 & 26,4 & 25,5 & 24,7 & 24,0 \\
\hline 0,28 & 61,0 & 52,8 & 47,4 & 43,3 & 40,2 & 37,7 & 35,5 & 33,7 & 32,1 & 30,8 & 29,5 & 28,4 & 27,5 & 26,6 & 25,7 & 25,0 \\
\hline 0,29 & 64,9 & 55,6 & 49,6 & 45,3 & 42,0 & 39,3 & 37,0 & 35,1 & 33,4 & 32,0 & 30,7 & 29,6 & 28,5 & 27,6 & 26,7 & 25,9 \\
\hline 0,3 & 69,6 & 58,6 & 52,0 & 47,3 & 43,8 & 40,9 & 38,5 & 36,5 & 34,8 & 33,2 & 31,9 & 30,7 & 29,6 & 28,6 & 27,7 & 26,9 \\
\hline 0,31 & 75,5 & 61,9 & 54,5 & 49,5 & 45,6 & 42,6 & 40,1 & 37,9 & 36,1 & 34,5 & 33,1 & 31,8 & 30,7 & 29,7 & 28,7 & 27,9 \\
\hline 0,32 & 88,2 & 65,5 & 57,2 & 51,7 & 47,5 & 44,3 & 41,6 & 39,4 & 37,5 & 35,8 & 34,3 & 33,0 & 31,8 & 30,7 & 29,7 & 28,8 \\
\hline 0,33 & & 69,8 & 60,1 & 54,0 & 49,5 & 46,1 & 43,2 & 40,9 & 38,8 & 37,1 & 35,5 & 34,1 & 32,9 & 31,8 & 30,8 & 29,8 \\
\hline 0,3 & & 75,3 & 63,3 & 56,5 & 51,6 & 47,9 & 44,9 & 42,4 & 40,3 & 38,4 & 36,8 & 35,3 & 34,0 & 32,9 & 31,8 & 30,8 \\
\hline 0,35 & & 84,6 & 66,8 & 59,1 & 53,8 & 49,8 & 46,6 & 43,9 & 41,7 & 39,7 & 38,0 & 36,5 & 35,2 & 34,0 & 32,9 & 31,8 \\
\hline 0,36 & & & 71,0 & 61,9 & 56,1 & 51,8 & 48,4 & 45,5 & 43,2 & 41,1 & 39,3 & 37,8 & 36,4 & 35,1 & 33,9 & 32,9 \\
\hline 0,37 & & & 76,4 & 65,1 & 58,6 & 53,8 & 50,2 & 47,2 & 44,7 & 42,5 & 40,7 & 39,0 & 37,5 & 36,2 & 35,0 & 33,9 \\
\hline 0,38 & & & 86,6 & 68,7 & 61,2 & 56,0 & 52,1 & 48,9 & 46,2 & 44,0 & 42,0 & 40,3 & 38,7 & 37,3 & 36,1 & 35,0 \\
\hline 0,39 & & & & 73,0 & 64,1 & 58,3 & 54,0 & 50,6 & 47,8 & 45,4 & 43,4 & 41,6 & 39,9 & 38,5 & 37,2 & 36,0 \\
\hline 0,4 & & & & 78,7 & 67,3 & 60,8 & 56,1 & 52,5 & 49,5 & 47,0 & 44,8 & 42,9 & 41,2 & 39,7 & 38,3 & 37,1 \\
\hline
\end{tabular}


2.04 templating software (HECTEC GmbH, Germany) was used by observers A and D whereas IMPAX Orthopaedic Suite (Agfa Healthcare, Mortsel, Belgium) was used by observers $\mathrm{B}$ and $\mathrm{C}$. The observers were not instructed to calibrate the images in the same manner.

Intra-observer reliability was estimated by mean error between two sets of measurements. Standard deviation was also calculated for the mean errors. Pearson's correlation coefficient was used to estimate the similarity of the measurements. Significance level was set to 0.05 . The first series of measurements for version and inclination from each observer was chosen for the interobserver analysis. Thereby we could add sensitivity to the inter-observer analysis instead of calculating the mean values of the two measurement series. BlandAltman plots for version and inclination were calculated between each observer. With four observers there are four paired plots. First they were drawn between A and $\mathrm{B}, \mathrm{A}$ and $\mathrm{C}$ and $\mathrm{B}$ and $\mathrm{C}$. Subsequent plots were drawn between $\mathrm{A}$ and $\mathrm{D}, \mathrm{B}$ and $\mathrm{D}$ and $\mathrm{C}$ and $\mathrm{D}$. These were drawn for both version and inclination. Mean error and 95\% limits of agreement were calculated between each observer as stated by Bland et al. [19]. Intraclass correlation coefficient (ICC) was used to analyse the variability between the observers. Statistical analysis was done with SPSS 17.0 software (SPSS Inc., Chicago, USA).

\section{Results}

Intra-observer correlations and mean errors are presented in Table 1. All correlations were statistically significant $(p<$ 0.05). Bland-Altman plots between observers for version are shown in Fig. 4 and for inclination in Fig. 5. Mean error for version between observers $\mathrm{A}$ and $\mathrm{B}$ was 0.2 degrees, between $\mathrm{A}$ and $\mathrm{C} 1.0$ degrees, between $\mathrm{A}$ and $\mathrm{D}-0.03$ degrees, between $\mathrm{B}$ and $\mathrm{C} 0.7$ degrees, between $\mathrm{B}$ and $\mathrm{D}$ 0.7 degrees and between $\mathrm{C}$ and $\mathrm{D}-1.0$ degrees. Mean errors for inclination were $-0.5,0.1,-0.2,0.6,0.4$ and -0.3 degrees in the same manner. ICC for version among observers $\mathrm{A}, \mathrm{B}$ and $\mathrm{C}$ were 0.88 and among all observers 0.79 . The same values for inclination were 0.93 and 0.91 .

\section{Discussion}

Computed tomography (CT) imaging is often considered as the golden standard in the determination of the version of the cup component in hip arthroplasty. When the version is calculated with $\mathrm{CT}$, it is calculated against a plane formed by anatomical landmarks and considered as true version. If this reference plane matches the coronal plane, in theory, there is no difference between measurements done with CT or using plain AP radiographs. However, if there is pelvic tilting, the McKibbin plane formed by anteriosuperior iliac spines and pubic symphysis, is not parallel to the coronal plane [20]. This may lead to large differences in acetabular versions obtained from plain radiographs when compared to measurements done by CT. It has been shown that with fixed anteversion the correlation between pelvic tilt and measured anteversion is linear [21]. Due to centering of the $\mathrm{X}$-ray beam to the pubic symphysis the acetabular cup component is imaged obliquely in the whole pelvis radiographs. As a result the cup opening is imagined obliquely. In CT the cup opening is seen directly in the anteroposterior direction and this causes differences in calculations assuming the parallelism of the coronal and McKibbin plane. Derbyshire described a way to estimate this error and to correct the calculated version [22]. Widmer on the other hand proposed a correction of the version by 5.46 degrees when the imaging distance is 1.15 metres [5]. Pradhan preferred a correction of 5 degrees [6].

Marx et al. compared measurements done with five different radiographic methods and CT. A method by Widmer produced 9-degree greater values than four other radiographic methods [5, 23]. The values calculated by methods other than Widmer's were within 0.2 degrees. The difference was probably due to a correction of 5.46 degrees which is included in the method by Widmer. The correction value is affected by the imaging distance which in Widmer's method is 1.15 metres. The difference between values done with Widmer's method and with CT was -6.4 degrees, ie. CT produced greater values. The difference between the other four methods and CT based measurements varied between -14.3 to -14.5 degrees. In the study of Mayr et al. the mean inclination of the pelvis or pelvic tilt in the standing position was 6.7 degrees among 120 patients when
Table 1 Intra-observer values for inclination and version

$S D$ standard deviation

\begin{tabular}{|c|c|c|c|c|}
\hline \multirow[t]{2}{*}{ Observer } & \multicolumn{2}{|l|}{ Version } & \multicolumn{2}{|l|}{ Inclination } \\
\hline & Mean error (SD) & Correlation & Mean error (SD) & Correlation \\
\hline A & $0.3(1.3)$ & 0.97 & $0.3(1.7)$ & 0.96 \\
\hline B & $-0.28(2.8)$ & 0.85 & $-0.1(2.4)$ & 0.97 \\
\hline $\mathrm{C}$ & $1.1(2.1)$ & 0.93 & $-0.4(1.8)$ & 0.97 \\
\hline $\mathrm{D}$ & $-1.8(3.1)$ & 0.74 & $-0.6(2.1)$ & 0.94 \\
\hline
\end{tabular}


Fig. 4 Bland-Altman plot for version measurements

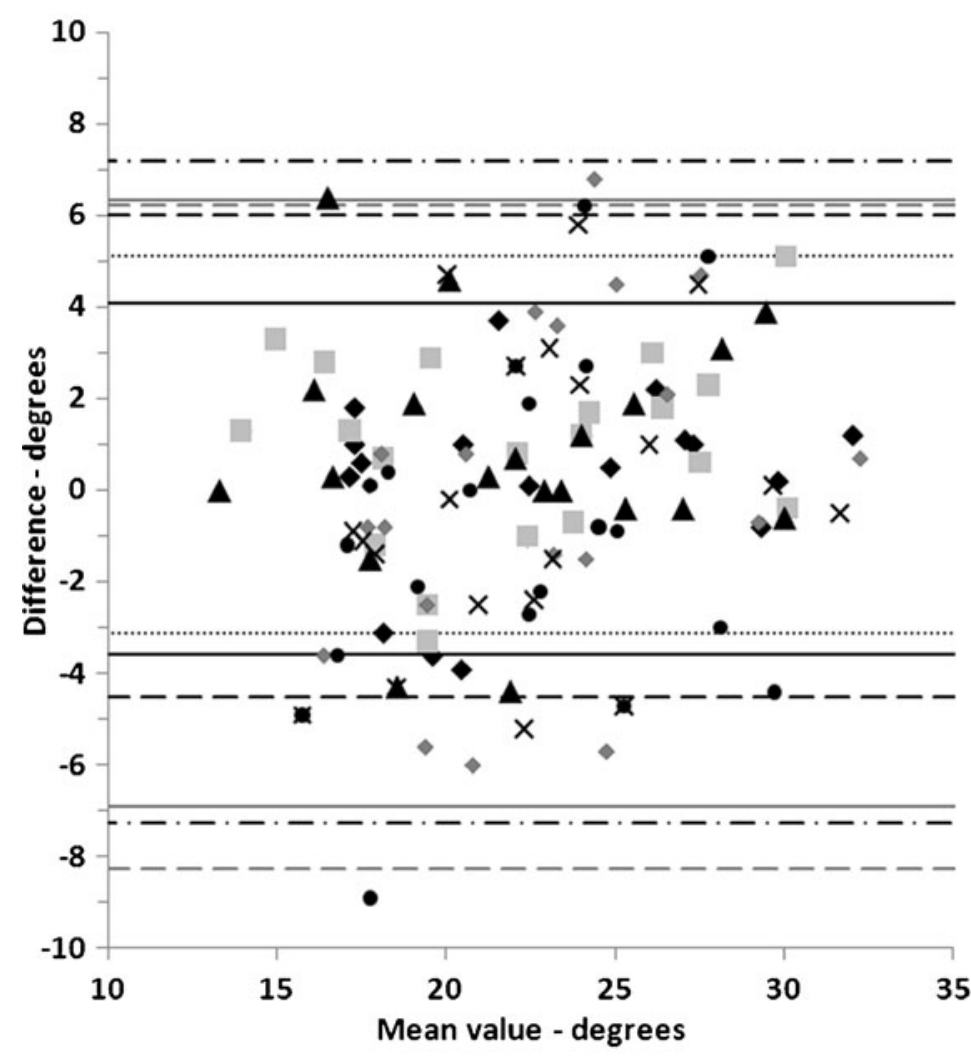

- Observer A and B

In Observer A and C

- Observer A and D

A Observer B and C

$\times$ Observer B and D

- Observer C and D

- Limits of agreement between $A$ and $B$

........ Limits of agreement between $A$ and $C$

- - - Limits of agreement between $A$ and $D$

- - Limits of agreement between $B$ and $C$

- Limits of agreement between $B$ and $D$

- - Limits of agreement between $\mathrm{C}$ and $\mathrm{D}$
McKibbin plane was used as the reference [24]. If the pelvic tilt of 6.7 degrees is corrected so that the McKibbin plane matches the coronal plane, the radiologically estimated cup version is increased by a value which can be precisely calculated as described by Murray [25]. If this is applied to the findings of Marx et al., the differences
Fig. 5 Bland-Altman plot for inclination measurements

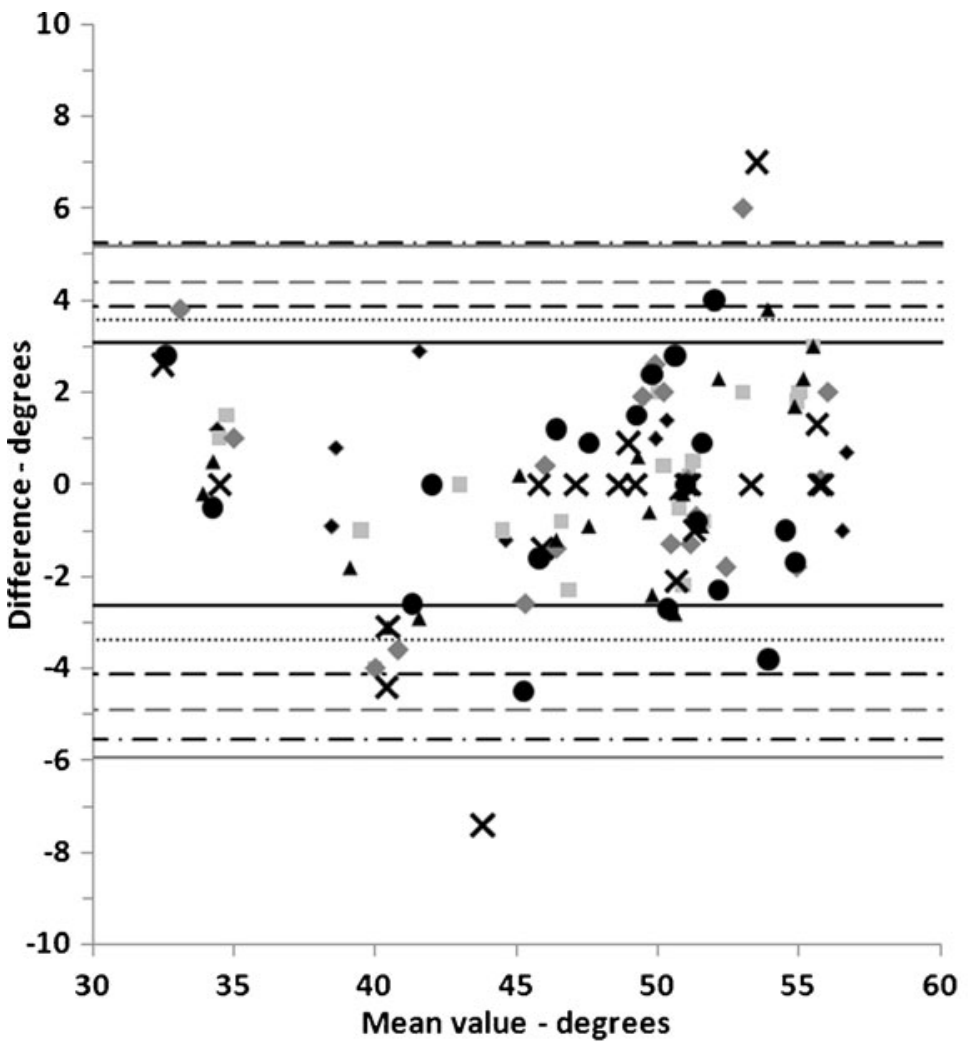

- Observer A and B

In Observer $\mathrm{A}$ and $\mathrm{C}$

- Observer A and D

- Observer B and C

X Observer B and D

- Observer C and D

L Limits of agreement between $A$ and $B$

......... Limits of agreement between $\mathrm{A}$ and $\mathrm{C}$

- - - Limits of agreement between $A$ and $D$

- - Limits of agreement between $B$ and $C$

- Limits of agreement between $B$ and $D$

- - Limits of agreement between $C$ and $D$ 
between radiographic and $\mathrm{CT}$ measurements and essentially decreased.

In conclusion there is not a consensus about the correct anatomical reference plane. In the study of Ghelman et al. the reference plane was formed by posterior border of the distal sacrum and the posterior border of the ischium [26]. Marx et al., Olicrona et al., and Kalteis et al. used the McKibbin plane as a reference $[23,27,28]$. It is obvious that these two different planes are not parallel in the vast majority of the population.

Thus the measurements done with plain radiographs or based on CT have different premises. The former defines the planar values and the latter true or anatomical values. In another words cup orientation respective to the coordinate system based on the bony hip can be measured with $\mathrm{CT}$ and spatial orientation of the cup is calculated from plain radiographs. The measured radiographic version can be considered as functional since that is the position against which the femoral component articulates.

Pelvic rotation and pelvic tilt are the major causes which produce inaccuracy in measurement of version between subsequent radiographs. Tannast et al. measured six different parameters to estimate pelvic tilt and rotation. The best correlation with pelvic tilt was 0.68 for men and 0.63 for women [29]. Most importantly the inaccuracy due to the pelvic tilt or pelvic rotation can be minimised by an organised and systematic imaging procedure. Imaging in the supine position should be avoided since the difference between the measured version in the supine and standing positions may be 20 degrees at most [30]. It should also be remembered that when using plain radiographs in the assessment of the cup orientation, a retroverted cup cannot be excluded by a simple AP radiograph. Care should be taken when interpreting the calculated version as anteversion.

The method presented by us is applicable to all cups which present as spherical contour. Unfortunately this method is not ideal for acetabular cups such as ASR (DePuy, Warsaw, Indiana, USA) which has nonspherical shape [31]. The quality of the radiographs and version of the cup dictates whether the double stepped outline of the ASR cup can be visualised or not [14]. With increased version this outline becomes less invisible and we recommend that calculations for the ASR prosthesis should be performed with the EBRA-system.

Previously, De Haan et al. applied the method of Widmer in their failure study of metal-on-metal resurfacings [15]. However, when using the method by Widmer the short axis of the ellipse of the cup opening needs to be visualised. In the case of all-metal prosthesis this must be approximated to a great extent. The axiolateral method may be easily used with MMHRs but the same issues, ie. finding the major axis of the ellipse, are related to it as when using AP radiographs. Also it should be noted that in the axiolateral radiographs the reference level or anteroposterior line is greatly affected by the patient's supine position.

Langton et al. studied the reliability of the EBRA system in the calculation of the cup version in MMRH [14]. Limits of agreement varied between -5 to +6 degrees among five observers compared to a reference value. Differences in measurements between observers in our study equals those using the EBRA system in the study of Langton et al. We did not however have a reference value to compare the measurements. However we strongly believe that the interand intra-observer errors were satisfactory and our research frame was adequate to study the repeatability and usefulness of our method.

Langton et al. also proposed a simple grading system to qualitatively assess the version of the cup. But as they state, in some cases it is not applicable (Fig. 1b, c, d). The major distinction in our method is that it can be applied in all possible angulations of the cup. In addition, inclination of the cup can be measured exactly since the major axis of the ellipse formed by the cup opening is drawn.

The method proposed by Hassan is compatible to use with all-metal prostheses as such. However, the most important thing in the determination of version is to find the end points of the major semi axis of the ellipse formed by the cup opening. It is the most important factor to cause inter-observer variability. This can be done accurately by drawing a circle continuous with the outline of the cup as proposed by us (Fig. 2).

In our study we found that limits of agreement between observer $\mathrm{D}$ and the others were higher when compared to ones among observers A, B and C. However, the mean errors in the same arrangement did not differ. We believe the differences in the limits of agreement were due to inexperience of the use of the templating software and the interpretation of the projection of the resurfacing prosthesis in the radiographs. Therefore, it is mandatory to be familiar with postoperative radiographs of hip arthroplasty and the software used when performing quantitative measurements.

In conclusion the mathematical method presented to assess the version and inclination of the cup in the metalon-metal bearing shows that the limits of agreement and the mean errors between observers were at an acceptable level. Also, this method may be performed with any orthopaedic templating software. The observations clearly demonstrated the necessity of mastering the technique as evinced in nonconforming results in one of the measurement series done without previous experience with the software used. This method is most applicable for routine clinical use with its ease of use and favourable cost implications.

Conflict of interest The authors declare that they have no conflict of interest. 


\section{References}

1. Malik A, Maheshwari A, Dorr LD (2007) Impingement with total hip replacement. J Bone J Surg [Am] 89:1832-1842

2. Triclot P (2011) Metal-on-metal: history, state of the art (2010). Int Orthop 35:201-206

3. De Haan R, Pattyn C, Gill HS, Murray DW, Campbell PA, De Smet K (2008) Correlation between inclination of the acetabular component and metal ion levels in metal-on-metal hip resurfacing replacement. J Bone J Surg [Br] 90:1291-1297

4. Langton DJ, Jameson SS, Joyce TJ, Webb J, Nargol AV (2008) The effect of component size and orientation on the concentrations of metal ions after resurfacing arthroplasty of the hip. $\mathrm{J}$ Bone $\mathrm{J}$ Surg [Br] 90:1143-1151

5. Widmer KH (2004) A simplified method to determine acetabular cup anteversion from plain radiographs. J Arthroplasty 19:387390

6. Pradhan R (1999) Planar anteversion of the acetabular cup as determined from plain anteroposterior radiographs. J Bone J Surg [Br] 81:431-435

7. Fabeck L, Farrokh D, Tolley M, Descamps PY, Gebhart M, Delince P (1999) A method to measure acetabular cup anteversion after total hip replacement. Acta Orthop Belg 65:485-491

8. Ackland MK, Bourne WB, Uhthoff HK (1986) Anteversion of the acetabular cup. Measurement of angle after total hip replacement. J Bone J Surg [Br] 68:409-413

9. Liaw CK, Hou SM, Yang RS, Wu TY, Fuh CS (2006) A new tool for measuring cup orientation in total hip arthroplasties from plain radiographs. Clin Orthop 451:134-139

10. Seradge H, Nagle KR, Miller RJ (1982) Analysis of version in the acetabular cup. Clin Orthop 166:152-157

11. Hassan DM, Johnston GH, Dust WN, Watson LG, Cassidy D (1995) Radiographic calculation of anteversion in acetabular prostheses. J Arthroplasty 10:369-372

12. Malviya A, Lingard EA, Malik A, Bowman R, Holland JP (2010) Hip flexion after Birmingham hip resurfacing: role of cup anteversion, anterior femoral head-neck offset, and head-neck ratio. J Arthroplasty 25:387-391

13. Langton DJ, Sprowson AP, Joyce TJ, Reed M, Carluke I, Partington P, Nargol AV (2009) Blood metal ion concentrations after hip resurfacing arthroplasty: a comparative study of articular surface replacement and Birmingham hip resurfacing arthroplasties. JBJS [Br] 91:1287-1295

14. Langton DJ, Sprowson AP, Mahadeva D, Bhatnagar S, Holland JP, Nargol AV (2010) Cup anteversion in hip resurfacing: validation of EBRA and the presentation of a simple clinical grading system. J Arthroplasty 25:607-613

15. De Haan R, Campbell PA, Su EP, De Smet KA (2008) Revision of metal-on-metal resurfacing arthroplasty of the hip: the influence of malpositioning of the components. J Bone $\mathrm{J}$ Surg [Br] 90:1158
16. Ball ST, Schmalzried TP (2009) Posterior femoroacetabular impingement (PFAI) after hip resurfacing arthroplasty. Bull NYU Hosp Jt Dis 67:173-176

17. Yao L, Yao J, Gold RH (1995) Measurement of acetabular version on the axiolateral radiograph. Clin Orthop 316:106-111

18. Reito A, Puolakka T, Pajamäki J (2010) Birmingham hip resurfacing: five to eight year results. Int Orthop. doi:10.1007/ s00264-010-1066-9, 19 June 2010 [Epub ahead of print]

19. Bland JM, Altman DG (1986) Statistical methods for assessing agreement between two methods of clinical measurement. Lancet 8476:307-310

20. McKibbin B (1970) Anatomical factors in the stability of the hip joint in the newborn. JBJS [Br] 52:148-159

21. Haenle M, Heitner A, Mittelmeier W, Barbano R, Scholz R, Steinhauser E, Bader R (2007) Assessment of cup position from plain radiographs: impact of pelvic tilting. Surg Radiol Anat 29:29-35

22. Derbyshire B (2008) Correction of acetabular cup orientation measurements for X-ray beam offset. Med Eng Phys 30:1119 1126

23. Marx A, von Knoch M, Pfortner J, Wiese M, Saxler G (2006) Misinterpretation of cup anteversion in total hip arthroplasty using planar radiography. Arch Orthop Trauma Surg 126:487-492

24. Mayr E, Kessler O, Prassl A, Rachbauer F, Krismer M, Nogler M (2005) The frontal pelvic plane provides a valid reference system for implantation of the acetabular cup: spatial orientation of the pelvis in different positions. Acta Orthop 76:848-853

25. Murray DW (1993) The definition and measurement of acetabular orientation. J Bone J Surg [Br] 75:228-232

26. Ghelman B, Kepler CK, Lyman S, Della Valle AG (2009) CT outperforms radiography for determination of acetabular cup version after THA. Clin Orthop 467:2362-2370

27. Kalteis T, Handel M, Herold T, Perlick L, Paetzel C, Grifka J (2006) Position of the acetabular cup - accuracy of radiographic calculation compared to CT-based measurement. Eur J Radiol 58:294-300

28. Olivecrona H, Weidenhielm L, Olivecrona L, Beckman MO, Stark A, Noz ME, Maguire GQ Jr, Zeleznik MP, Svensson L, Jonson T (2004) A new CT method for measuring cup orientation after total hip arthroplasty: a study of 10 patients. Acta Orthop Scand $75: 252-260$

29. Tannast M, Murphy SB, Langlotz F, Anderson SE, Siebenrock KA (2006) Estimation of pelvic tilt on anteroposterior X-rays - a comparison of six parameters. Skeletal Radiol 35:149-155

30. Eddine TA, Migaud H, Chantelot C, Cotten A, Fontaine C, Duquennoy A (2001) Variations of pelvic anteversion in the lying and standing positions: analysis of 24 control subjects and implications for CT measurement of position of a prosthetic cup. Surg Radiol Anat 23:105-110

31. Shimmin A (2010) The influence of the size of the component on the outcome of resurfacing arthroplasty of the hip. J Bone J Surg [Br] 92:469-476 